\title{
Recent results of the cold tests performed on the stellarator W7-X coils
}

\author{
J. Baldzuhn, H. Ehmler, A. Hoelting, C. Sborchia, K. Hertel, L. Genini ${ }^{1}$, T.Schild ${ }^{1}$ \\ Max-Planck Institut fuer Plasmaphysik IPP, EURATOM Association, \\ 17491 Greifswald, Germany \\ (1) CEA/DSM/DAPNIA, Centre de Saclay, 91191 Gif-sur-Yvette, France
}

\begin{abstract}
The magnetic plasma fusion experiment Wendelstein 7-X (W7-X) will use a system of superconducting coils for the production of the magnetic field. In the first paragraph, W7-X is described very briefly, with emphasis on the coil system. These superconducting coils are tested thoroughly in a test facility. The test procedure is described in the second paragraph. In the third paragraph, some test results are described in more detail. Focus is here on the measurement of mechanical stresses during cool-down and application of an electrical current to the coils. We describe the measurement of the electrical impedance to detect short-circuits. Finally, we present the quench tests which are used investigate the superconductor quality, and the Paschen tests used for the check of the electrical coil surface insulation.
\end{abstract}

\section{THE STELLARATOR W7-X}

At present, the fusion experiment Wendelstein 7-X [1] is under construction at the MaxPlanck-Institute for Plasma Physics (IPP) in Greifswald, Germany. Aim of this experiment is the demonstration of a net-current free fusion relevant plasma operation close to steady-state conditions, i.e. magnetically confined plasma discharges with a duration of up to 30 minutes. Superconducting coils are mandatory for discharges of that length. The superconducting coil system of W7-X consists of 50 non-planar and 20 planar coils, which will produce a magnetic flux density of up to $3 \mathrm{~T}$ in the plasma centre. The total plasma volume will be about $32 \mathrm{~m}^{3}$, the average radius of the non-planar coils is about $1.25 \mathrm{~m}$. The magnetic flux density at the coils will be more than $6 \mathrm{~T}$. W7-X will be a toroidal arrangement in the form of a pentagon, where five straight parts (the five modules) are connected by five corners. The average diameter of the pentagon will be $5.5 \mathrm{~m}$.

The coils will be cooled with supercritical helium at a temperature of $\approx 3-4 \mathrm{~K}$ with an inlet pressure of $0.6 \mathrm{MPa}$ [2]. Each non-planar coil consists of 108 windings of an internally cooled CICC superconducting cable (Cable-in-Conduit Conductor), each planar coil consists of 36 windings [3]. Those cables contain 243 individual superconducting strands, lying in an aluminium jacket with a void fraction of $37 \%$. Between the strands, the cooling helium is flowing. Each strand is made of $160 \mathrm{NbTi}$ filaments, embedded in a copper matrix. The outer jacket cross section is $16 \mathrm{X} 16 \mathrm{~mm}$.

Each winding pack consists of several layers, which are connected by interlayer joints. On each of those joints, quench detection (qd) wires are connected to allow for voltage drop measurements during the coil operation. Thus, the resistive voltage drop can be used to trigger a fast current ramp-down of the W7-X coil arrangement in the case of a quench. To avoid measured voltage changes due to externally induced magnetic flux changes, always two adjacent winding layers are electrically connected (compensated) to an electronic Wheatstone half-bridge arrangement.

In W7-X, always ten coils will be connected in series. In case of a fast current ramp-down, the voltage drop due to self-inductance across these ten coils will be in the range of symmetric \pm $3.5 \mathrm{kV}$ with respect to ground, with the grounding point between the coils no. 5 and 6 . Therefore, the qd wires have to be high-voltage proof. Particular attention is put on the failure case of a vacuum leak in the cryostat with a subsequent pressure increase and a fast current 
ramp-down. Paschen-tests on all coils are therefore performed to guarantee the high-voltage proof even under conditions close to the Paschen minimum.

Each winding pack is embedded with a mixture of quartz sand and epoxy into a stainless steel casing with an average wall thickness of $25 \mathrm{~mm}$. During the bedding procedure, the winding pack (having room temperature) is placed into the coil casing, having a temperature of about $120^{\circ} \mathrm{C}$. Thus, it should be guaranteed that during all operating conditions and coil currents the winding pack is firmly confined by the casing. Even with a current applied, the winding pack will never move inside the casing and can, thus, never provoke a quench. Additionally, the casing will always bear the major fraction of the mechanical forces acting on the winding pack. All 70 coils are situated in a toroidal cryostat, including the mechanical support structures, the $70 \mathrm{~K}$ shield, the vacuum pumping system, the superconducting current bus-bar system and current feed-throughs [4,5].

Each coil casing carries on the surface a system of helium cooling pipes being thermally connected to the casing material by copper stripes. Thus, each coil casing can be cooled independently from the winding pack. On each coil surface are attached several PT100 and CERNOX temperature sensors, as well as four strain gauges. The strain gauges consist of 3element rosettes in a 0-45-90 degrees arrangement. The measurement taps of two rosettes are combined in an electronic Wheatstone half-bridge arrangement, one rosette being glued to the casing surface, and one to a stainless steel compensation block. Thus, any apparent strain, induced for instance by a change of the electrical resistance with the temperature, is compensated. Always two strain gauges are placed adjacent to each other, providing redundant measurement values. To measure the mechanical extension of the entire coil, steel wires are clamped across the coil, acting on displacement potentiometers. Unfortunately, no sensors or gauges are available inside the superconducting winding pack.

The W7-X coil system will be used to produce a stellarator magnetic field configuration. In such a stellarator, no net plasma current is flowing (in opposite to the tokamak arrangement, realised for instance for ITER). The complete magnetic configuration is produced in a stellarator by the external coil system alone. The plasma is confined inside a concentric magnetic flux bundle of nested closed magnetic surfaces. Each flux surface is enclosed by a single magnetic vector field line of infinite length. To sustain such a complex magnetic topology, the magnetic field strength and vector orientation in space must have a relative accuracy of better $10^{-4}$. This requires a very high precision during the coil production. The deformation of the coils during operation due to mechanical and magnetic forces has to be minimised by employing sophisticated bearing and supporting techniques.

Furthermore, once the cryostat will be closed, no further access to the coils will be possible during the entire period of operation. Any misalignment or deformation of the coils will then have a severe and permanent impact on the magnetic topology. The only means of correction might then be the use of additional external coils. Any reparation or maintenance after a coil damage will be almost impossible, for instance if a high-voltage breakthrough occurs. Therefore, all coils are subject to intense and thorough tests, as they are described below. For each test result, pre-defined specifications and minimum or maximum values are fixed and have to be fulfilled. According to a prescribed procedure, all test results are recorded, stored and documented. Some results are used for later evaluations and investigations, for instance for the quench behaviour, or for the assessment of the mechanical stresses.

\section{COIL TEST PROCEDURE}

All coil tests are performed at the Commissariat a l'Energie Atomique (CEA) in Saclay, France. Two vacuum tanks are available there, each of them large enough to accept two coils simultaneously. A cold facility provides liquid nitrogen and helium to cool down the coils, and a regulated high-current power supply allows to energise the coils with the nominal 
current (16 kA for the planar and $17.6 \mathrm{kA}$ for the non-planar coils). To minimise the testing time, several coils can be tested simultaneously.

After the coil arrival in Saclay, a first visual check is performed to detect damages by transportation. A check of integrity of all sensors and qd-wires is performed to guarantee correct operation. A dc current of $20 \mathrm{~A}$ is applied to the coil to measure the cable and the joint resistance. Then, a dc high-voltage is applied to the coils $(6.3 \mathrm{kV}$ on the planar and $9.1 \mathrm{kV}$ on the non-planar coils) to measure the leak current. The overall resistance has to be larger than $500 \mathrm{M} \Omega$. Then the hydraulic gas flow impedance is measured, both for the casing cooling tubes and the winding pack.

After that incoming inspection, the coils are installed in a test support. Together with that support they are then installed in a vacuum tank, where the sensor checks and the dc highvoltage test is repeated. In addition, an ac high-voltage test is performed at $1.4 \mathrm{kV}$ (peak-topeak) to measure the high-voltage proof between the conductor terminations of the coils. After closing the vacuum tank and pumping down, both the casing tubes and the winding pack are pressurised with helium, and the helium tightness of the complete arrangement is measured. The maximum allowed integral leak-rate is $10^{-5} \mathrm{~Pa} \cdot 1 / \mathrm{s}$. Then the dc high-voltage test is repeated once again under vacuum conditions.

If all preceding tests were successful, the cooling down of the coils is started. During that period, all sensor signals (temperatures, mechanical stresses in the casing, vacuum leak rate, deformation of the casing, helium inlet and outlet pressures, helium flow rate, winding pack resistance) are recorded to allow for a later assessment of the cooling down behaviour. Once the final temperature of about $5 \mathrm{~K}$ is reached, the dc and ac high-voltage tests are repeated. Under stationary thermal and hydraulic conditions, the liquid helium flow impedance of both the casing tubes and the winding pack are measured at different helium pressure drops.

As the coils are now superconducting, the nominal current is applied at nominal temperature (5.8 $\mathrm{K}$ for the non-planar and $7.1 \mathrm{~K}$ for the planar coils) and all sensor signals are recorded and evaluated. No external magnetic field can be applied, the coils are tested in their selffield. If stationary conditions are maintained, the helium inlet temperature is increased in steps of $100 \mathrm{mK}$. After each step, stationary conditions have to be maintained again, then the next step follows until a quench occurs. After the quench, the coils are tested again with nominal current at nominal temperature to make sure that no damages occured.

After the successful quench test, the coils are warmed up, and the dc and ac high-voltage tests, as well as the hydraulic flow measurements are repeated. Finally, a Paschen-test is performed. For this test, all blank metallic surfaces facing the vacuum are carefully insulated, if they are electrically connected to the winding pack and the qd-wires. Then a dc high-voltage is applied to the winding pack, simultaneously the vacuum background pressure is increased in steps from good vacuum (better than $10^{-2} \mathrm{~Pa}$ ) up to atmospheric pressure. The background gas is air. Under all circumstances, the electric leak resistance has to be larger than $500 \mathrm{M} \Omega$. By means of video cameras, flashes or electric break-throughs can be recorded. Focus is on the pressure range between $10 \mathrm{~Pa}$ and $100 \mathrm{~Pa}$, where the Paschen break-through voltage minimum is expected.

\section{SOME TEST RESULTS}

All test results are recorded and documented. In particular all measured time traces are provided as ascii-files, to allow for a later evaluation of selected data or the investigation of interesting aspects. In the following, we focus on the more detailed description of four topics: the investigation of the mechanical stresses, the electrical impedance measurements, the quench tests and the Paschen-tests.

As mentioned above, up to four strain gauge rosettes are attached to the coil surface. From the strain gauge data, the stresses in the coil casing are calculated, either as a function of the coil temperature or as a function of the coil current. This is of importance for the assessment of the 
bedding procedure. The strain gauge signals are evaluated to find the principal stresses, the van-Mises stresses and the principal stress angles.

The most fundamental question concerns the development of the principal stresses as a function of the casing temperature, in particular for the comparison between the redundant sensors. For some of the sensors, the measurements revealed obviously a not adequate gluing of the gauge stripes on the carrier materials. This information helped to improve the gluing procedure and initiated the continued improvement of the process of sensor application.

For the assessment of the bedding procedure, the dependence of the stresses from the coil current $\mathrm{I}^{2}$ is evaluated, which should be linear. It is found that a roughly linear relation is given for all coils under investigation, so far. However, a more detailed analysis shows that a least squares fit to a polynomial function turns out as slightly better than a linear fit. The reason for that slight deviation from a linear relation is not yet understood. It is suspected that either the bedding procedure might not provide the desired result to the full extent, or that again not appropriate application of the sensors might play a role.

The fig. 1 shows, as an example, the measured principal stresses versus the coil current $\mathrm{I}^{2}$. Obviously, it is not possible to fit the entire curves to one linear function. Much more, the slope of the curves changes at $\mathrm{I} \approx 11 \mathrm{kA}$, possibly indicating a re-organisation of the confining forces on the winding pack at this current value.

During the ac high-voltage tests of the coils, in parallel the complex impedance of the winding pack is evaluated as a function of the applied current frequency. The impedance is determined by a stray capacity between winding and casing in the range of several $10 \mathrm{nF}$, and by the fact that any current change in the winding pack will induce a current in the casing. Thus, the coils act as transformers with one secondary winding. To understand better the electric behaviour of this RLC network, analytical and numerical models of the coils are developed and compared to the measurements. This is of particular interest, if possible shortcircuits inside the winding pack are searched. It was found, both by measurement and calculation, that the coil impedance changes strongly if a short-circuit is present. In particular, a pronounced resonance frequency at about $20 \mathrm{kHz}$ (for the non-planar coils) is predicted and measured. The value of that resonance frequency changes strongly in the case of a shortcircuit. However, the sensitivity of that method is rather limited for the case, that no "hard" short-circuit exists, but only an inter-winding current leak at higher electrical resistance. Fig. 2 shows the comparison of the measured and calculated absolute value of the complex impedance $\mathrm{Z}$ for the non-planar coils. Very good agreement is given for frequencies below 20 $\mathrm{kHz}$, as well as for the location of the resonance frequency.

To investigate the high-voltage proof of the coil insulation under any background pressure conditions, Paschen-tests are performed. Those tests are presently done at CEA, but preparing Paschen-tests are also performed by the coil manufacturers, and in the future they will be performed directly before coil installation at IPP. By now, it turned out that the perfect surface insulation of the coils is very difficult to maintain. In particular the expoxy sleeves around the interlayer joints suffer from Paschen defects during the tests. This can be explained by the fact that they are hand-made, and a perfect covering of the complicated shape of the joints is difficult to realise. Vacuum impregnated expoxy sleeves, as they are also applied to the coils, normally do not suffer from those defects. In order to improve that situation, additional formed parts were developed which are attached to the joints. Thus, the hazard of discharges close to the Paschen-minimum could strongly be reduced. Fig. 3 shows a photograph taken during a Paschen-test on a planar coil. Shown are two interlayer joints, with a discharge between the right-hand joint to the coil casing.

An essential aspect during the tests is the performance of temperature quenches. Each coil is operated at nominal temperature (about $0.3 \mathrm{~K}$ below the expected quench temperature) and nominal current in the coil self-field. Then the helium inlet temperature is increased in small steps $(0.1 \mathrm{~K})$ until a quench occurs. By now, all coils investigated so far showed a measured 
quench temperature which was, on the average, about $0.2-0.3 \mathrm{~K}$ higher than the specification, indicating a very good quality of the superconductors. Comparing the time traces of the essential parameters during the quench indicates an almost identical behaviour of all coils.

Fig. 4 shows some measured time traces during a time interval of about 12 hours for such a quench test. The inlet helium temperature (solid line) is increased in small steps until a quench is reached (at about 18:00 h). The outlet temperature (dashed line) increases synchronically, always being about $0.6 \mathrm{~K}$ higher than the inlet temperature. This results from the heat load from the coil and the resistive heat from the electrical joints. The current (dotted line) is first ramped up and down several times to test the stability of the arrangement, and remains then at $17.6 \mathrm{kA}$ until the quench is reached.

Fig. 5 shows some measured time traces during a time interval of about 3 seconds directly before and after a quench. The quench occurs at about $0.9 \mathrm{~s}$. The compensated resistive voltage (dotted line) starts to increase at $\approx 0.55 \mathrm{~s}$, indicating the initiation of the quench. At $\approx$ $0.7 \mathrm{~s}$ the total resistive voltage drop reaches $500 \mathrm{mV}$ and triggers the quench alarm threshold. After a time delay of about $150 \mathrm{~ms}$, the high current switches are activated. They start the current ramp-down (dashed line) with an average time constant of $2.2 \mathrm{~s}$. Simultaneously to the current ramp-down, the induced coil voltage (over one layer) reaches a maximum value of about 110 Volt (solid line) at the beginning and decays then exponentially, too. The typical induced voltage drops over the entire coil are about $400 \mathrm{~V}$ in the test facility. As expected, all quenches observed so far were initiated on the high-field side layers of the coils.

\section{PRESENT STATUS}

Until today, 9 coils out of 70 were tested successfully. In general, the quench behaviour of the coils is very good and the superconductor performance is even slightly better than specified. For all coils, the measured quench temperatures were higher than the specified values.

Several defects on the coils could be detected and repaired. Most severe defects were caused by vacuum leaks on the casing cooling tubes resulting from aggressive soldering flux. A revision of the soldering procedure eliminated that hazard. It was also found that the qd-wires were not high-voltage proof and had to be replaced. Now, the complete cabling is highvoltage tight. The coil surface insulation layers turned at the beginning out as not Paschenproof, but that problem could also be fixed by re-work of the insulation according to a revised procedure.

Very frequently, damaged or badly cabled sensors or gauges were found. Even though those errors are not essential, their detection is cumbersome and time consuming. Nevertheless, the coil testing procedure became in the meantime a matter of routine, providing a maximum of reliability for the coils to be installed in W7-X.

\section{References}

[1] G. Grieger et al, Phys. Fluids B 4 (1992) page 2081

H.J. Hartfuss et al, "Advanced Diagnostics for Magnetic and Interial Fusion", Eds.: P.E.Stott, A. Wooton. Kluwer, New York NY 2002, page 371

[2] M. Nagel, F. Schauer, IEEE Trans. Appl. Supercond. 12 (2002) page 1537

[3] T. Schild et al, IEEE Trans. Appl. Supercond. 13, (2003) page 1468 T. Schild et al, Proc. MT-19 Conf. Geneva (2001)

[4] Technische Spezifikation für das Nichtplanare Spulensystem des W7-X Experimentes, IPP technical document No.: AAB-S03.1

[5] X. Cheng, W. Lehmann, FZ Karlsruhe Report FZKA5517 (1995) 
Figure captions

Fig 1: Principal stresses (sigma) versus the squared coil current $\mathrm{I}^{2}$. The first principal stress is shown as dots, the second as circles. The solid lines show linear fits to the measured data.

Fig 2: Measured (dots) and calculated (solid line) absolute value of the complex impedance Z versus frequency.

Fig 3: Photograph of a discharge during an unsuccessful Paschen-test. The arrows indicate the start and end of the discharge.

Fig 4: Time traces of the coil current (dotted line, right y-axis), the helium inlet temperature (solid line, left y-axis) and the helium outlet temperature (dashed line, left y-axis) versus time. The experiment start at 9:00 am. and ends with a quench at 18:20 pm.

Fig 5: Time traces of the coil current (dashed line, left y-axis), total voltage drop across one coil layer (solid line, right y-axis), and compensated voltage (dotted line, right y-axis, voltage multiplied by 100) versus time. The quench is initiated at about $0.5 \mathrm{~s}$, the quench alarm system triggers the current switches at about $0.7 \mathrm{~s}$, the current decay starts at about $0.9 \mathrm{~s}$. 


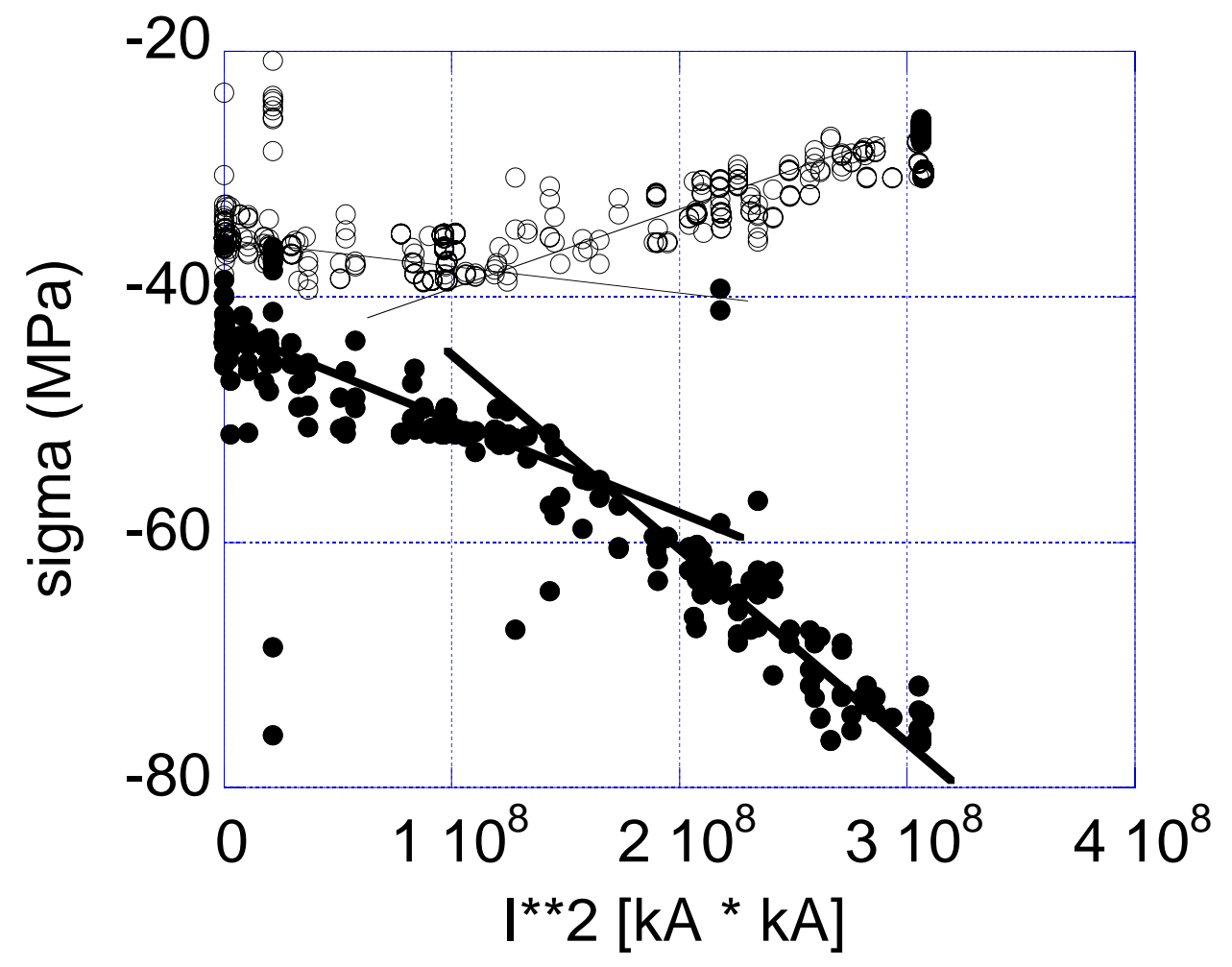




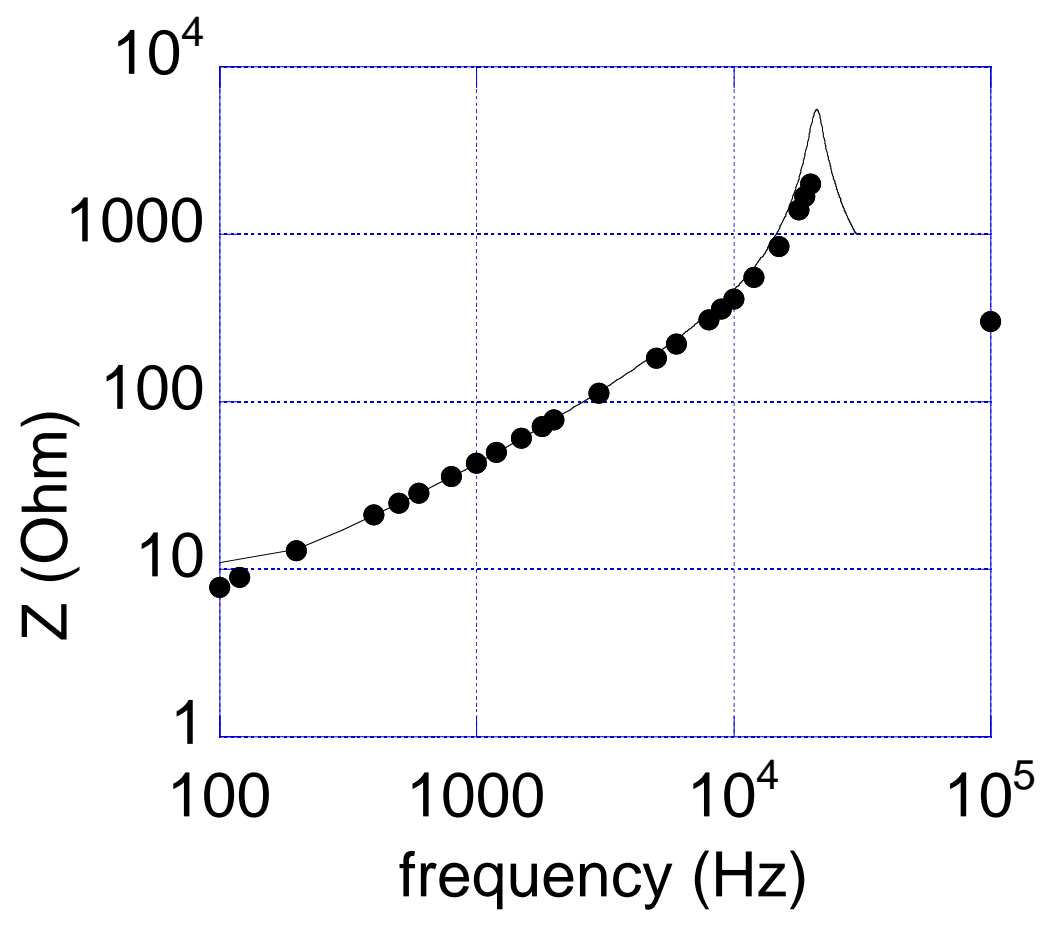




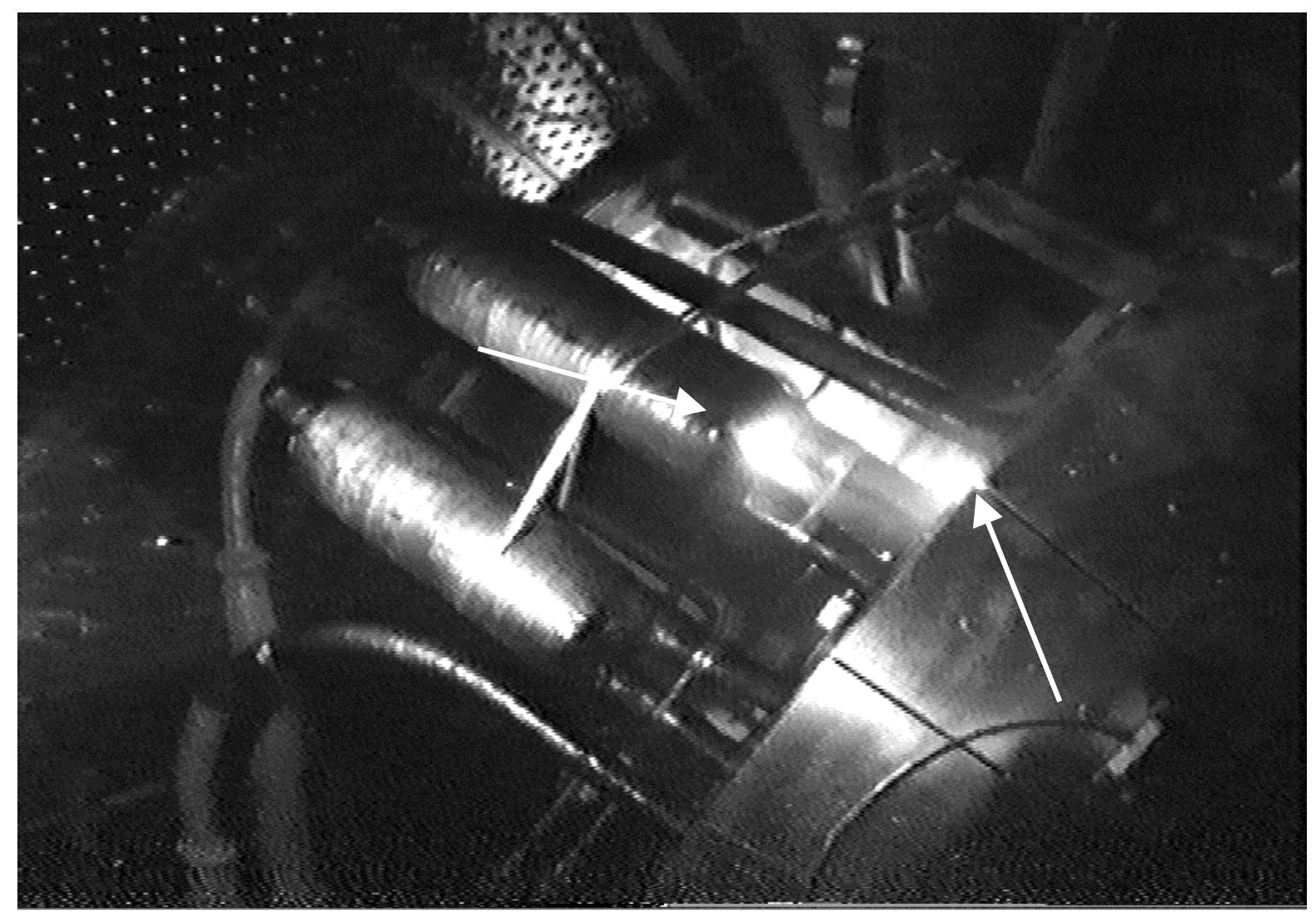




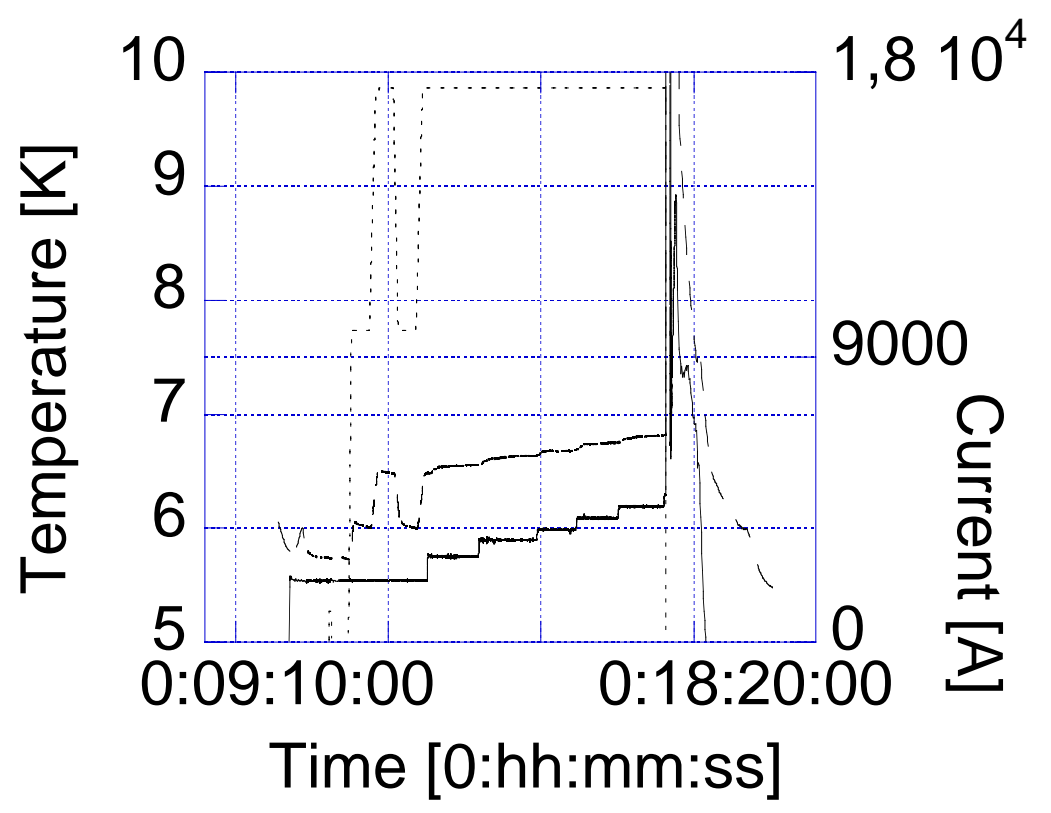




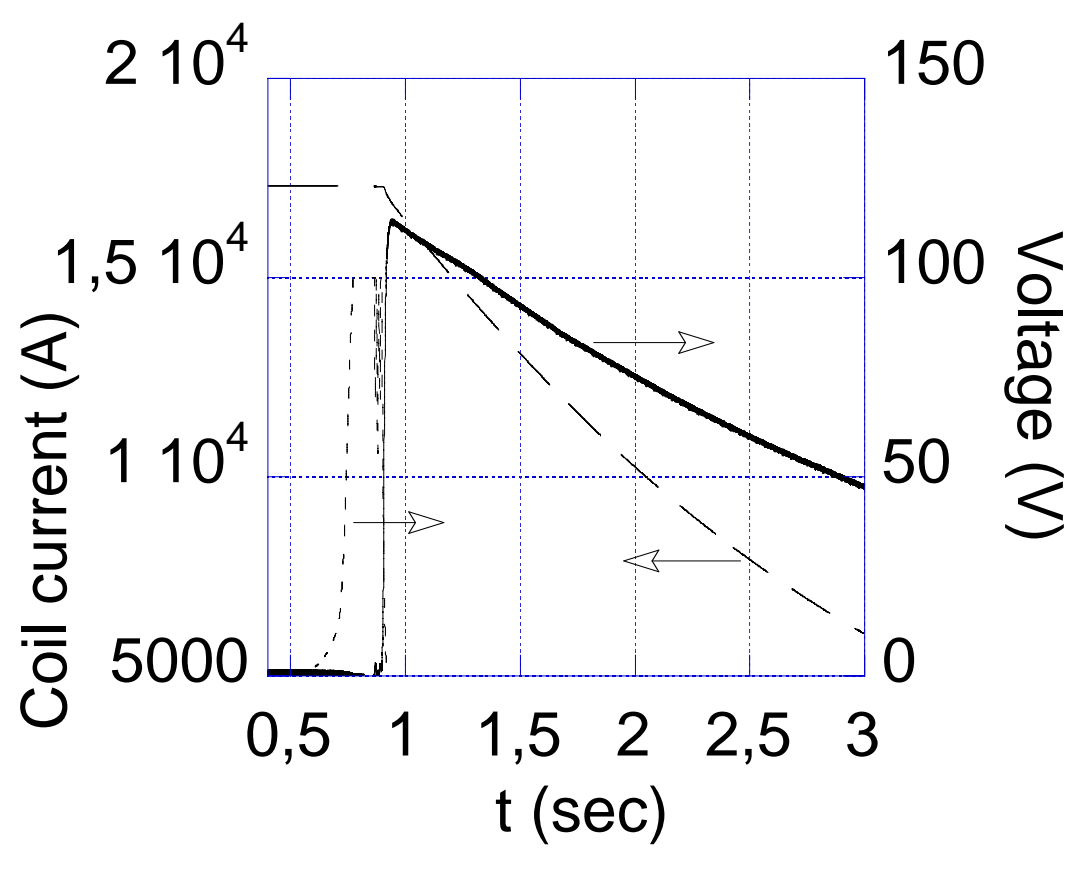

